\title{
Radiological localization of greater palatine foramen using multiple anatomical landmarks
}

\begin{abstract}
Identification of greater palatine foramen is of prime value for dentists and the oral and maxillofacial surgeons. The objective of present study was to radiologically localize greater palatine foramen with multiple anatomical landmarks. All Computer Tomography scans of individuals who have undergone paranasal sinus evaluation were obtained from the Department of Radiology, Azeezia Institute of Medical Sciences, from April 2015 to April 2016. Distance of greater palatine foramen from various known anatomical landmarks was measured across the CT slices. Forty-four CT scans were studied, mean age was $32( \pm 2.3)$ years. All scans were from individuals of south Indian origin. GPF was located at $38.38 \mathrm{~mm}$ from incisive fossa, $17.6 \mathrm{~mm}$ from posterior nasal spine, $18.38 \mathrm{~mm}$ from intermaxillary suture, $5.03 \mathrm{~mm}$ from second molar and $5.28 \mathrm{~mm}$ from third molar. Distances of GPF from incisive foramen and intermaxillary suture differed significantly on right and left sides. In $25(56.8 \%)$ cases GPF was located closer to third molar. In seven cases, it was closer to second molar and in 12 cases, GPF was located at the junction of second and third molar. Posterior location of GPF, posterior to third molar is not noted. The utilization of multiple anatomical reference points, such as the incisive foramen, the intermaxillary suture, and the second and third molars simplifies identification of GPF.
\end{abstract}

Keywords: greater palatine foramen, inter maxillary suture, second molar, third molar, ct scan
Volume 2 Issue 7 - 2016

\author{
Viveka S,' Mohan Kumar² \\ 'Department of Anatomy,Azeezia Institute of Medical Sciences, \\ India \\ ${ }^{2}$ Department of Radiology,Azeezia Institute of Medical Sciences, \\ India
}

Correspondence: Viveka S, Assistant professor, Department of Anatomy, Azeezia Institute of Medical Sciences, Kollam, India, Email vivekabharathi@gmail.com

Received: May 25, 2016 | Published: December 29, 2016
Abbreviations: GPF, greater palatine foramen; IF, incisive fossa; PNS, posterior nasal spine; IMS, intermaxillary suture; M2, second molar; M3, third molar

\section{Introduction}

Greater palatine foramen (GPF) and canal contains greater palatine nerve and vessels. Greater palatine nerve is a branch of maxillary division of trigeminal nerve supplying mucosa of hard palate, medial wall of maxillary sinus and posterior aspect of lateral wall of nose. Identification of GPF is of prime value for dentists and the oral and maxillofacial surgeons. There are two techniques of GPF block, high tuberosity approach and greater palatine approach. ${ }^{1,2}$ The former procedure involves injection of anaesthetic in pterygopalatine fossa from highest point on the mucobuccal fold just distal to the second maxillary molar teeth to achieve block of maxillary division of trigeminal nerve. ${ }^{3}$ The later technique is commonly used. Three dimensional orientation of GPF is important for successful local anaesthesia. ${ }^{4}$ GPF is located approximately at $1 \mathrm{~cm}$ medial and adjacent to second molar teeth and with a cotton swab is pressed to detect a relative defect produced by the foramen. ${ }^{5}$ Exact location of GPF and direction of greater palatine canal varies. There are reported variations of greater palatine nerve within the canal. ${ }^{6}$ There are many studies reporting location and direction of foramen and canal in various ethnic groups. Many such studies are dry bone studies.

There are many studies reporting morphology and variations of GPF in dry skulls of individuals of foreign origin..$^{7-10}$ It is appreciated that there is renewed interest among Indian researchers in the recent past. Nidhi Sharma has studied one hundred dry human adult skulls and defined GPF morphology in north Indian population. ${ }^{2}$ Mitesh Dave et al. ${ }^{11}$ have studied another hundred skulls of Gujarat origin. Saralaya et al. ${ }^{12}$ have studied 132 skulls from west costal region of
India. Sathya Priya et al. ${ }^{13}$ \& Murali P et al. ${ }^{14}$ have studied another 132 and 137 skulls of Tamil Nadu region respectively. Ashwini et al. ${ }^{15}$ have reported data from 100 north Karnataka skulls. Vaibhav Anjankar et al. ${ }^{16}$ have studied 86 skull data from central India. Ajay Kumar et al. ${ }^{17}$ have reported measurements from 100 skulls of Punjab origin. GPF morphology and variations are also subjected to significant scrutiny using come beam computed tomography. ${ }^{18-21}$ Morphological studies are done with regular CT scans. ${ }^{22}$ Such studies of GPF from Indian subcontinent are sparse. No other Indian study has radiologically studied GPF in our subcontinent to our knowledge. Limited literature is available for radiological landmarks for localization of GPF location in Indian population. The objective of this study was to define the location of GPF using multiple anatomical landmarks.

\section{Methodology}

All Paranasal air sinuses Computer Tomography (CT) scans of individuals who have undergone CT scan of head for purpose of para nasal sinus evaluation were obtained from the Department of Radiology, Azeezia Institute of Medical Sciences, from April 2015 to April 2016. At the beginning, institutional ethics committee cleared the study.

\section{Inclusion criteria}

Individuals with fully erupted third molars on both sides of the maxilla, presence of all maxillary teeth, patient age of $>21$ years were evaluated in the present study.

\section{Exclusion criteria}

Patients who had pathology of para nasal air sinuses; nose and nasopharynx were excluded from the study in order to negate possible distorted anatomy of GPF. 


\section{Images}

A high-speed GE FX CT scanner (GE Healthcare, India) acquired CT images at exposure $120 \mathrm{kV}, 74 \mathrm{~mA}, 60 \mathrm{mAs}$; rotation time 0.5 ; slice thickness $0 . \mathrm{mm}$. Patient's sex and age data were acquired from patient files. Image evaluation was under three planes-coronal, sagittal and transverse. Linear and compound measuring tool measured the distances. Two authors measured the distances twice individually at two different occasions. Final values were average of the observations. This minimised the inter-observer variations. Lowest count of measuring tool was $0.1 \mathrm{~mm}$.

\section{Measurement}

Measurements of the distance between the GPF and the incisive foramen (IF), the posterior nasal spine (PNS), the intermaxillary suture (IMS), as well as the second (M2) and third (M3) molars was done. In the axial plane, after identification of GPF, distance between the incisulare was measured. The centre of the GPF was established while measuring its anterior-posterior (AP) and transverse dimensions. The centre of the GPF was set at the point of the intersection of two straight lines representing the longest AP and transverse GPF dimensions.
Location and distance of GPF from lateral border of molars noted next at higher axial slice. Distances from intermaxillary suture and from PNS were noted at still higher planes having GPF point fixed. The data was tabulated and analysed using MS-excel software. Comparisons were by student $\mathrm{t}$ test and values $<0.05$ was considered significant.

\section{Results}

From the department of Radiology, forty-four CT scans were included for the study. Out of which 30 were males and 14 were females. Mean age was $32( \pm 2.3)$ years. All scans were from individuals of south Indian origin. GPF was located at $38.38 \mathrm{~mm}$ from incisive fossa, $17.6 \mathrm{~mm}$ from posterior nasal spine (PNS), $18.38 \mathrm{~mm}$ from intermaxillary suture, $5.03 \mathrm{~mm}$ from second molar and $5.28 \mathrm{~mm}$ from third molar. Measurements on right and left sides are tabulated in Table 1. Distances of GPF from incisive foramen and intermaxillary suture differed significantly on right and left sides. No such significant changes noted in distances from molars. In $25(56.8 \%)$ cases GPF was located closer to third molar. In seven cases, it was closer to second molar and in 12 cases, GPF was located at the junction of second and third molar. Posterior location of GPF, posterior to third molar is not noted (Figure 1).

Table I Measurements of distances of greater foramen from incisice fossa (IL), posterior nasal spine (PNS), Intermaxillary suture (IMS), second molar (M2) and third molar (M3) on right (R) and left $(\mathrm{L})$ sides. All measurements in millimetres $(\mathrm{mm})$; SD- standard deviation and SE- standard error; $\mathrm{n}=44$ cases; $\nmid \mathrm{P}<0.05$

\begin{tabular}{llllllllllll}
\hline & IF(R) & IF(L) & PNS(R) & PNS(L) & IMS(R) & IMS(L) & M2(R) & M2(L) & M3(L) & M3(L) \\
\hline Mean & 39.76 & $37 \dagger$ & 17.78 & 17.44 & 16.72 & $20.03 \dagger$ & 4.93 & 5.13 & 4.85 & 5.71 & \\
SD & 2.47 & 2.39 & 1.78 & 2.02 & 2.61 & 3.38 & 1.57 & 2.69 & 1.54 & 2.11 & 0.61 \\
SE & 0.87 & 0.98 & 0.49 & 0.58 & 0.75 & 1.38 & 0.45 & 0.81 & 0.46 & 6.2 \\
Mode & 43 & 37 & 18.2 & 16 & 20.5 & 20 & 6.4 & 2.2 & 5.7 & 6.2 & 6.3 \\
Median & 39.25 & 36.55 & 17.8 & 17.15 & 16.45 & 20.5 & 5.3 & 6.5 & 5.2 & 2.3 \\
Min & 36.7 & 34.8 & 14 & 14.8 & 12.1 & 13.1 & 2.2 & 1.6 & 2.5 & 2.3 \\
Max & 43 & 42 & 21.5 & 21.8 & 20.5 & 23.9 & 7.8 & 8.5 & 7.9 & 8.5 \\
\hline
\end{tabular}

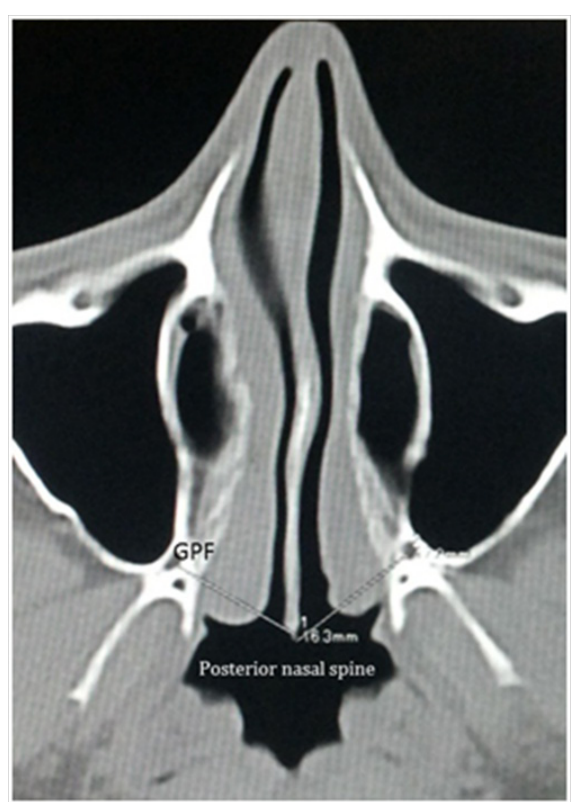

Figure I Measurement of distance from greater palatine foramen (GPF) to posterior nasal spine (blue arrow); point of greater palatine foramen is fixed and at a lower slice of CT scan other distances are measured.

\section{Discussion}

Greater palatine canal approach to maxillary nerve block, demands a perfect three-dimensional orientation of position, direction and extent of the canal. The preliminary step is identification of GPF. Utilizing multiple anatomical landmarks to identify the GPF increases the accuracy and minimizes the complications of injecting anaesthetic drug. In numerous previous studies using dry adult skulls, there is more emphasis on all the measurements of present study. However, our study, being radiological evaluation, the location of GPF is more accurate than the dry bone studies. Our findings of distances of GPF from incisive foramen, posterior nasal spine and intermaxillary suture correlates with dry bone studies. ${ }^{2,9-12,15}$ Our findings are reflective of values from cone-beam studies of GPF. ${ }^{18,20,21}$ Position of GPF in this study was noted next to third molar in $56 \%$, whereas many previous studies have reported $70-90 \%$ in relation to third molar. ${ }^{7,22,23,24}$ This may be attributed to fewer subjected in the present study.

The location of GPF in relation to third molar is consistent with anaesthetic procedure. ${ }^{3,5}$ In most procedural explanation, it is stated that GPF is located around $5 \mathrm{~mm}$ from lateral wall of third molar or second molar. Through this study, we have also noted that there is no statistically significant difference between right and left side. Of particular note, in one of the cases, GPF was located very close $(1.2 \mathrm{~mm})$ from third molar. Authors are of the opinion, that distance 
of GPF to pterygoid hamulus, posterior border of hard palate, edge of soft palate and pterygomaxillary suture that is reported widely in the previous dry bone studies are not of critical clinical significance. As these points (pterygoid hamulus, posterior border of hard palate, pterygomaxillary suture) cannot be localized in a patient with certainty. Touching the soft palate or adjacent lateral pharyngeal wall, elicit a gag reflex. A major limitation of the current study is we have considered only forty-four scans. Most dry bone studies and cone beam GPF studies comments on the direction of greater palatine canal. We have not commented on the direction and length of the canal in the present study.

\section{Conclusion}

The utilization of multiple anatomical reference points, such as the incisive foramen, the intermaxillary suture, and the second and third molars simplifies identification of GPF. This in turn simplifies the task of greater palatine approach anaesthesia and reduced complications.

\section{Acknowledgements}

None.

\section{Conflict of interest}

Author declares that there is no conflict of interest.

\section{References}

1. Aoun G, Zaarour I, Sokhn S, et al. Maxillary nerve block via the greater palatine canal: An old technique revisited. J Int Soc Prev Community Dent. 2015;5(5):359-364.

2. Varshney R, Ray S, Sharma N. Anatomic and anesthetic considerations of greater palatine nerve block in Indian population. Saudi J Med Med Sci. 2014;2(3):197-201.

3. Baheti DK, Bakshi S, Gupta S, et al. Interventional Pain Management: A Practical Approach. JP Medical Ltd; 2016:542.

4. Kang SH, Byun IY, Kim JH, et al. Three-Dimensional Analysis of Maxillary Anatomic Landmarks for Greater Palatine Nerve Block Anesthesia. J Craniofac Surg. 2012;23(3):e199-e202.

5. Malamed SF. Handbook of Local Anesthesia. Amsterdam, Netherlands: Elsevier Health Sciences; 2014. p. 435.

6. Hafeez NS, Ganapathy S, Sondekoppam R, et al. Anatomical variations of the greater palatine nerve in the greater palatine canal. $J$ Can Dent Assoc. 2015;81:f14.

7. Ilayperuma I, Nanayakkara G, Palahepitiya N. Morphometric evaluation of the greater palatine foramen in adult Sri lankan skulls. Int J Morphol. 2014;32(4):1418-1422.

8. Nimigean V, Nimigean VR, Buţincu L, et al. Anatomical and clinica considerations regarding the greater palatine foramen. Rom J Morphol Embryol. 2013;54(3 Suppl):779-783
9. Soto RA, Caceres F, Vera C. Morphometry of the greater palatal canal in adult skulls. J Craniofac Surg. 2015;26(5):1697-1699.

10. Chrcanovic BR, Custódio ALN. Anatomical variation in the position of the greater palatine foramen. J Oral Sci. 2010;52(1):109-113.

11. Mitesh R Dave, Vaishali Kiran Yagain, Samir Anadkat. A study of the anatomical variations in the position of the greater palatine foramen in adult human skulls and its clinical significance. Int $J$ Morphol. 2013;31(2):578-583.

12. Saralaya V, Nayak SR. The relative position of the greater palatine foramen in dry Indian skulls. Singapore Med J. 2007;48(12):1143-1146.

13. Sathya Priya B, WMS Johnson, Lakshmanan P, et al. The relative position of greater palatine foramen in dried human adult unsexed skull bones. Res J Pharm Biol Chem Sci. 2015;6(5):869-876.

14. Murali P, Sundarapandian S, Madhan Kumar SJ. Relative anatomica position of greater palatine foramen with reference to intraoral landmarks in south Indian population. Int J Anat Res. 2016;4(1):1912-1915.

15. Ashwini $\mathrm{H}$, Jaishree $\mathrm{H}$. The morphometric study of greater palatine foramen in dry adult skulls. Indian $\mathrm{J}$ Med Case Reports. 2014;3(4):73-77.

16. Vaibhav P Anjankar, Gupta SD, Shema Nair, et al. Analysis of position of greater palatine foramen in central Indian adult skulls: a consideration for maxillary nerve block - ProQuest. Indian J Pharm Biol Res. 2014;2(1):51-54.

17. Ajay Kumar, Anu Sharma, Poonam Singh. Assessment of the relative location of greater palatine foramen in adult Indian skulls: Consideration for maxillary nerve block. Eur J Anat. 2011;15(3):150-154.

18. Ikuta CRS, Cardoso CL, Ferreira Júnior O, et al. Position of the greater palatine foramen: an anatomical study through cone beam computed tomography images. Surg Radiol Anat. 2013;35(9):837-842.

19. Monsour P, Huang TC. Morphology of the greater palatine grooves of the hard palate: A CBCT study. Aust Dent J. 2015;61(3):329-332.

20. Rapado González O, Suárez Quintanilla JA, Otero Cepeda XL, et al. Morphometric study of the greater palatine canal: cone-beam computed tomography. Surg Radiol Anat. 2015;37(10):1217-1224.

21. Yilmaz HG, Boke F, Ayali A. Cone-beam computed tomography evaluation of the soft tissue thickness and greater palatine foramen location in the palate. J Clin Periodontol. 2015;42(5):458-461.

22. Tomaszewska IM, Kmiotek EK, Pena IZ, et al. Computed tomography morphometric analysis of the greater palatine canal: a study of 1,500 head CT scans and a systematic review of literature. Anat Sci Int. 2014;90(4):287-297.

23. Jaffar AA, Hamadah HJ. An analysis of the position of the greater palatine foramen. J Basic Med Sci. 2003;3(1):24-32.

24. Methathrathip D, Apinhasmit W, Chompoopong S, et al. Anatomy of greater palatine foramen and can and pterygopalatine fossa in Thais: considerations for maxillary nerve block. Surg Radiol Anat. 2005;27(6):511-516. 